\title{
NÂNG CAO Độ CHÍNH XÁC CỦA MÔ HÌNH QUASIGEOID QUỐC GIA NHỜ XÂY DỤ๋NG HỆ QUY CHIẾU KHÔNG GIAN QUỐC GIA
}

\author{
HÀ MINH HÒA \\ Viện Khoa học Đo đạc và Bản đồ
}

\section{Tóm tắt:}

Trong quá trình xây dụng hệ quy chiếu không gian quốc gia, trên cở sở định vị lại ellipsoid WGS84 quy chiếu quốc gia sát nhất với mặt quasigeoid quốc gia, chúng ta sẽ nâng cao độ chính xác của 07 tham số chuyển tọa độ tù ITRF về hệ quy chiếu không gian quốc gia, đặc biêt là tham số thay đổi tỷ lẹ xích giữa hai hệ tọa đô nêu trên. Điều này cho phép chuyển đổi chính xác độ cao trắc địa toàn cầu được xác định bằng công nghệ GNSS tù ellipsoid WGS84 quy chiếu quốc tế về ellipsoid WGS84 quy chiếu quốc gia phục vu việc xây dụng mô hình quasigeoid quốc gia độ chinh xác cao. Bài báo khoa học này sẽ luận chứng cho cách tiếp cận nêu trên.

\section{1. Đặt vấn đề}

Đối với điểm GNSS bất kỳ, giả sử điểm $\mathrm{P}$, sau khi xử lý các dữ liệu GNSS trong ITRF, chúng ta nhận được các tọa độ trắc địa toàn cầu $\bar{B}, \bar{L}, \bar{H}$ của nó tương ứng với ellipsoid WGS84 quy chiếu quốc tế, ở đây độ cao trắc địa toàn cầu $\bar{H}$ là độ cao của điểm $\mathrm{P}$ so với mặt ellipsoid WGS84 quy chiếu quốc tế (xem Hình 1).

Giả thiết rằng hệ quy chiếu không gian quốc gia được xây dựng tương ứng với ellipsoid WGS84 quy chiếu quốc gia, thêm vào đó ellipsoid WGS84 quy chiếu quốc gia nhận được từ các kết quả định vị ellipsoid WGS84 quy chiếu quốc tế sát nhất với mặt quasigeoid cục bộ tại trạm nghiệm triều 0 (ở Việt Nam, trạm nghiệm triều 0 là trạm nghiệm triều Hòn Dấu). Lưu ý rằng mặt quasigeoid cục bộ trùng với mặt geoid cục bộ và sát nhất với mặt biển trung bình nhiều năm tại trạm nghiệm triều 0 , thêm vào đó mặt quasigeoid cục bộ nêu trên là mặt khởi tính cho hệ độ cao chuẩn quốc gia. Điều này có nghĩa là độ cao chuẩn quốc gia $H^{\gamma}$ của điểm $\mathrm{P}$ là độ cao của điểm này so với mặt quasigeoid cục bộ (xem Hình 1).

Nếu tồn tại 07 tham số chuyển các tọa độ $d X_{0}, d Y_{0}, d Z_{0}, \varepsilon_{X}, \varepsilon_{Y}, \varepsilon_{Z}, \Delta m$ từ ellipsoid WGS84 quy chiếu quốc tế về ellipsoid WGS84 quy chiếu quốc gia, các tọa độ trắc địa quốc gia (cục bộ) $B, L, H$ của điểm $\mathrm{P}$ tương ứng với ellipsoid WGS84 quy chiếu quốc gia, thêm vào đó độ cao trắc địa cục bộ $H$ là độ cao của điểm $\mathrm{P}$ so với mặt ellipsoid WGS84 quy chiếu quốc gia (xem Hình 1), sẽ được xác định theo các công thức sau:

$$
\begin{aligned}
& \left(\begin{array}{l}
B \\
L
\end{array}\right)=\left(\begin{array}{l}
\bar{B} \\
\bar{L}
\end{array}\right)+\left[\begin{array}{ccc}
-\frac{\sin \bar{B} \cdot \cos \bar{L}}{\bar{M}+\bar{H}} & -\frac{\sin \bar{B} \cdot \sin \bar{L}}{\bar{M}+\bar{H}} & \frac{\cos \bar{B}}{M+\bar{H}} \\
-\frac{\sec \bar{B} \cdot \sin \bar{L}}{\bar{N}+\bar{H}} & \frac{\sec \bar{B} \cdot \cos \bar{L}}{\bar{N}+\bar{H}} & 0
\end{array}\right] \cdot\left(\begin{array}{l}
d X_{0} \\
d Y_{0} \\
d Z_{0}
\end{array}\right)+ \\
& +\left[\begin{array}{llll}
-\left(1+e^{2} \cdot \cos 2 \bar{B}\right) \cdot \sin \bar{L} & \left(1+e^{2} \cdot \cos 2 \bar{B}\right) \cdot \cos \bar{L} & 0 & -e^{2} \cdot \sin \bar{B} \cdot \cos \bar{B} \\
\left(1-e^{2}\right) \cdot \operatorname{tg} \bar{B} \cdot \cos \bar{L} & \left(1-e^{2}\right) \cdot \operatorname{tg} \bar{B} \cdot \sin \bar{L} & -1 & 0
\end{array}\right] \cdot\left(\begin{array}{l}
\varepsilon_{X} \\
\varepsilon_{Y} \\
\varepsilon_{Z} \\
\Delta m
\end{array}\right)
\end{aligned}
$$




$$
H=\bar{H}+\cos \bar{B} \cdot \cos \bar{L} \cdot d X_{0}+\cos \bar{B} \cdot \sin \bar{L} \cdot d Y_{0}+\sin \bar{B} \cdot d Z_{0}+\Delta H\left(\varepsilon_{X}, \varepsilon_{Y}, \varepsilon_{Z}, \Delta m\right),
$$

ở đây thành phần

$$
\Delta H\left(\varepsilon_{X}, \varepsilon_{Y}, \varepsilon_{Z}, \Delta m\right)=\frac{1}{2} \cdot e^{2} \cdot \bar{N} \cdot \sin 2 \bar{B} \cdot\left(\cos \bar{L} \cdot \varepsilon_{Y}-\sin \bar{L} \cdot \varepsilon_{X}\right)+\left(\frac{a^{2}}{\bar{N}}+\bar{H}\right) \cdot \Delta m .
$$

Bài toán định vị ellipsoid WGS84 quy chiếu quốc tế sát nhất với mặt quasigeoid cục bộ tại trạm nghiệm triều 0 được thực hiện như sau. Trên tập hợp $\mathrm{n}$ điểm tham gia định vị có các độ cao chuẩn hạng I, II quốc gia $H_{i}^{\gamma}(i=1,2, \ldots, n)$ và các tọa độ trắc địa toàn cầu $\bar{B}_{i}, \bar{L}_{i}, \bar{H}_{i}(i=1,2, \ldots, n)$ nhận được từ các kết quả xử lý các dữ liệu GNSS trong ITRF tương ứng với ellipsoid WGS84 quy chiếu quốc tế.

Dựa trên nguyên tắc định vị ellipsoid sao cho các trục của ellipsoid WGS84 quy chiếu quốc gia song song với các trục tương ứng của ellipsoid WGS84 quy chiếu quốc tế, lưu ý thành phần $\Delta H\left(\varepsilon_{X}, \varepsilon_{Y}, \varepsilon_{Z}, \Delta m\right)$ (3) được nhận bằng 0 , công thức (2) có dạng:

$$
H=\bar{H}+A \cdot\left(\begin{array}{c}
d X_{0} \\
d Y_{0} \\
d Z_{0}
\end{array}\right),
$$

ở đây ma trận - hàng $\quad A=(\cos \bar{B} \cdot \cos \bar{L} \quad \cos \bar{B} \cdot \sin \bar{L} \quad \sin \bar{B})$.

Nếu điểm tham gia định vị $\mathrm{P}$ có độ cao chuẩn quốc gia $H^{\gamma}$ thì từ công thức (4) chúng ta nhận được công thức đối với quasigeoid quốc gia tại điểm $\mathrm{P}$ ở dạng sau:

$$
\zeta=\bar{\zeta}+A \cdot\left(\begin{array}{l}
d X_{0} \\
d Y_{0} \\
d Z_{0}
\end{array}\right),
$$

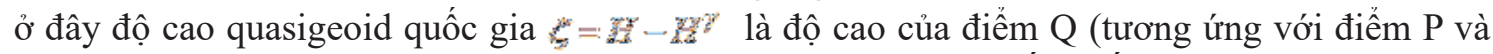
nằm trên mặt quasigeoid cục bộ) so với mặt ellipsoid WGS84 quy chiếu quốc gia, độ cao quasigeoid hỗn hợp $\overline{\bar{\xi}}=\overline{\mathbb{Z}}-\mathscr{Y}$ là độ cao của điểm $\mathrm{Q}$ so với mặt ellipsoid WGS84 quy chiếu quốc tế (xem Hình 1).

Điều kiện định vị ellipsoid sát nhất với mặt quasigeoid cục bộ có dạng $\sum_{i=1}^{n} \zeta_{i}^{2}=\min$. Nếu điểm tham gia định vị $\mathrm{P}$ là điểm độ cao nhà nước có đo GNSS, thì độ cao quasigeoid hỗn hợp $\overline{\bar{\xi}}=\overline{\mathbb{Z}}-M^{\gamma}$ trong phương trình (5) không có gì khác chính là độ cao quasigeoid GNSS/thủy chuẩn. Trên $\mathrm{n}$ điểm tham gia định vì là các điểm độ cao hạng I, II quốc gia có đo GNSS và xử lý các dữ liệu GNSS trong ITRF, sau khi giải hệ phương trình dạng (5) dưới điều kiện $\sum_{i=1}^{n} \xi_{i}^{2}=$ min. chúng ta sẽ nhận được các tham số chuyển tọa độ $d X_{0}, d Y_{0}, d Z_{0}$, thêm vào đó chúng là các tọa độ của tâm ellipsoid WGS84 quốc tế trong hệ quy chiếu tọa độ không gian quốc gia.

Theo tài liệu (Hà Minh Hòa, Nguyễn Ngọc Lâu, 2013), khi xử lý các dữ liệu GNSS trong ITRF với việc sử dụng lịch vệ tinh chính xác ở mức $M_{S}$ (đơn vị $\mathrm{cm}$ ), độ chính xác của độ cao trắc địa toàn cầu $\bar{H}$ được đánh giá theo công thức: $m_{H}= \pm \frac{M_{S}}{\sqrt{3}} \cdot \sec B$. 


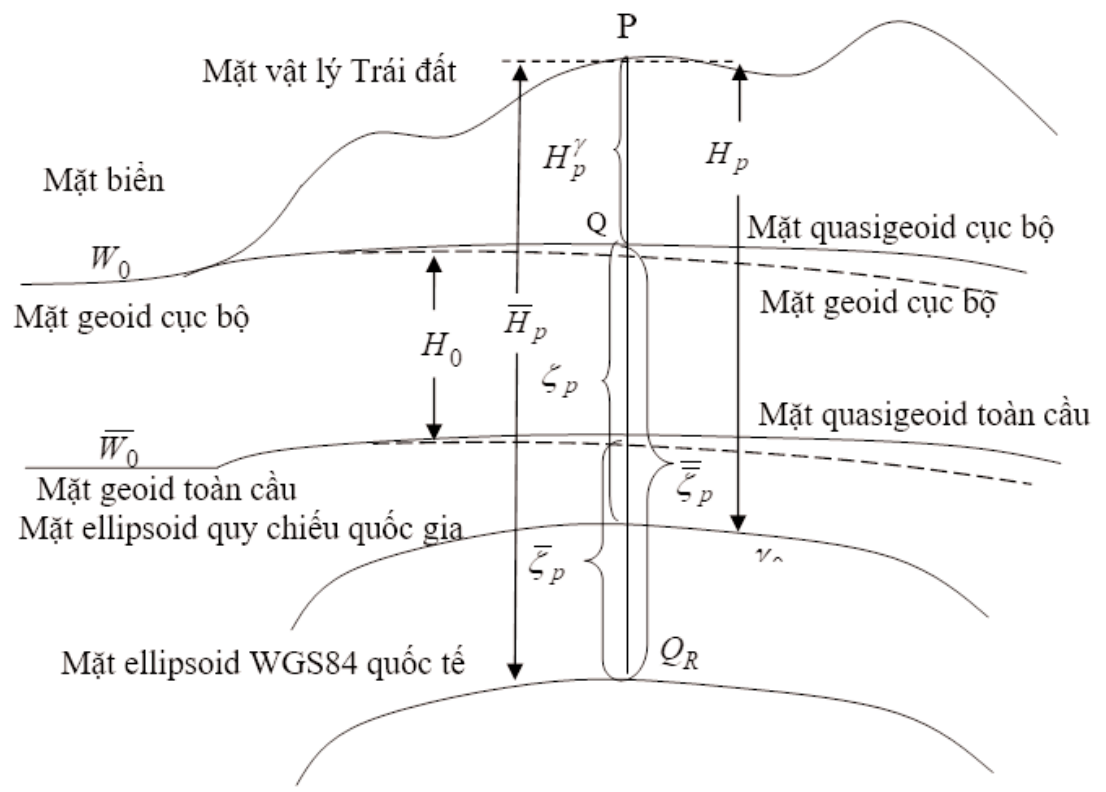

Hình 1: Các độ cao trắc địa, độ cao quasigeoid, độ cao chuẩn tuoong ứng với các mặt trong các hệ quy chiếu không gian quốc tế và quốc gia

Khi sử dụng lịch vệ tinh chính xác ở mức $M_{S}= \pm 2,5 \mathrm{~cm}$ thì độ cao trắc địa toàn cầu $\bar{H}$ có độ

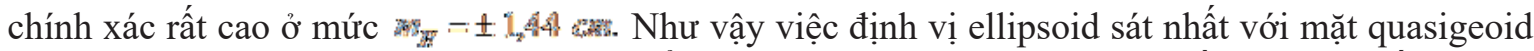
cục bộ và việc xác định các tham số chuyển tọa độ $d X_{0}, d Y_{0}, d Z_{0}$ từ ITRF về hệ quy chiếu không gian quốc gia không chỉ đảm bảo cho việc chuyển độ cao trắc địa toàn cầu $\bar{H}$ có độ chính xác rất cao thành độ cao trắc địa cục bộ $\mathrm{H}$ độ chính xác cao trong hệ quy chiếu không gian quốc gia theo công thức (4), mà còn đảm bảo cho việc chuyển độ cao quasigeoid hỗn hợp $\zeta$ chính xác thành độ cao quasigeoid quốc gia $\zeta$ theo công thức (5) trong hệ quy chiếu không gian quốc gia.

Chúng ta nhấn mạnh thêm rằng đối với $\mathrm{n}$ điểm tham gia định vị là các điểm độ cao hạng $\mathrm{I}, \mathrm{II}$ ổn định (không bị xê dịch bởi các tác nhân nhân sinh và tự nhiên) và có đo GNSS, các giá trị độ cao quasigeoid hỗn hợp $\bar{\zeta}$ có độ chính xác cao. Do đó từ công thức (5) chúng ta sẽ nhận được dãy bao gồm $\mathrm{n}$ giá trị độ cao quasigeoid quốc gia $\zeta$ độ chính xác cao trên $\mathrm{n}$ điểm độ cao hạng I, II ổn định, thêm vào đó dãy các giá trị độ cao quasigeoid quốc gia $\zeta$ hoàn toàn độc lập với các giá trị độ cao quasigeoid được xác định từ mô hình EGM2008-WGS84. Đây là cơ sở khoa học để đánh giá các giá trị độ cao quasigeoid được xác định từ mô hình EGM2008-WGS84 dựa trên dãy các giá trị độ cao quasigeoid quốc gia $\zeta$ nhận được từ các kết quả định vị ellipsoid.

Các độ cao trắc địa, độ cao quasigeoid, độ cao chuẩn trong các công thức được trình bày ở trên đều được chuyển về hệ triều 0 theo Nghị quyết No. 16 của Đại Hội đồng XVIII của IAG tại Hamburg (Đức) vào tháng 8 năm 1983 nhằm loại bỏ các sai số hệ thống được gây ra do hiệu ứng triều thường trực trong các đại lượng nêu trên.

Tuy nhiên, trong kỷ nguyên bình sai mạng lưới thiên văn - trắc địa quốc gia kết hợp với các dữ liệu GNSS, chúng ta luôn gặp nghịch lý (paradox). Từ các kết quả định vị ellipsoid, các tọa độ trắc địa $B_{0}, L_{0}$ của điểm gốc $\mathrm{O}$ của hệ tọa độ phẳng quốc gia được xác định theo công thức: 


$$
\left(\begin{array}{l}
B_{0} \\
L_{0}
\end{array}\right)=\left(\begin{array}{l}
\bar{B}_{O} \\
\bar{L}_{0}
\end{array}\right)+\left[\begin{array}{ccc}
-\frac{\sin \bar{B}_{O} \cdot \cos \bar{L}_{O}}{\bar{M}_{O}+\bar{H}_{O}} & -\frac{\sin \bar{B}_{O} \cdot \sin \bar{L}_{O}}{\bar{M}_{O}+\bar{H}_{O}} & \frac{\cos \bar{L}_{O}}{\bar{M}_{O}+\bar{H}_{O}} \\
-\frac{\sec \bar{B}_{O} \cdot \sin \bar{L}_{O}}{\bar{N}_{O}+\bar{H}_{O}} & \frac{\sec \bar{B}_{O} \cdot \cos \bar{L}_{O}}{\bar{N}_{O}+\bar{H}_{O}} & 0
\end{array}\right] \cdot\left(\begin{array}{l}
d X_{0} \\
d Y_{0} \\
d Z_{0}
\end{array}\right),
$$

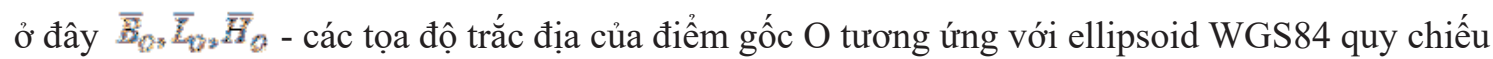
quốc tế và nhận được từ các kết quả xử lý các dữ liệu GNSS trên điểm $\mathrm{O}$ trong ITRF; $\overline{\mathrm{TH}_{\text {i }}}$ - bán kính cong trên kinh tuyến của điểm $\mathrm{O} ; \bar{N}$ - bán kính cong trên đường thẳng đứng thứ nhất của điểm $\mathrm{O}$.

Khi truyền các tọa độ trắc địa $B_{0}, L_{0}$ (hoặc các tọa độ phẳng $x_{0}, y_{0}$ ) của điểm gốc $\mathrm{O}$ ra toàn mạng lưới thiên văn - trắc địa hạng I, II quốc gia thông qua các trị đo thiên văn - trắc địa (góc, cạnh, cạnh đáy, phương vị Laplace), do sự tích lũy các sai số của các trị đo thiên văn - trắc địa, nên các tọa độ khái lược của các điểm thiên văn - trắc địa càng nằm xa điểm gốc $\mathrm{O}$ càng chứa các sai số lớn. Điều này làm nẩy sinh các tham số $\varepsilon_{X}, \varepsilon_{Y}, \varepsilon_{Z}, \Delta m$ trong các công thức (1) và (2). Các tham số này gây

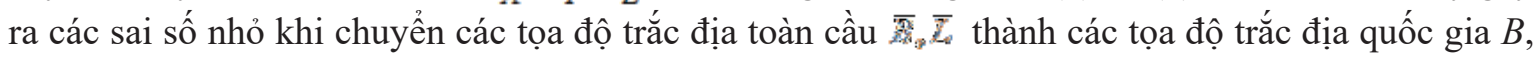
$L$ theo công thức (1). Ở Việt Nam, lượng thay đổi tỷ lệ xích $\Delta m=-0,000000252906278$ chỉ gây ra sự biến thiên trong giá trị vĩ độ trắc địa ở mức 0,0001285 " (ở vĩ độ trắc địa $\mathrm{B}=24^{\circ}$ ). Tuy nhiên, khi chuyển độ cao trắc địa toàn cầu $\bar{H}$ thành độ cao trắc địa quốc gia $H$ theo công thức (2), mặc dù các

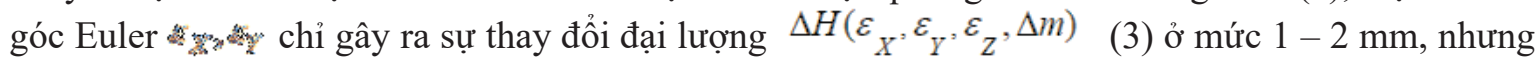
đại lượng thay đổi tỷ lệ xích $\Delta m$ có thể làm đại lượng $\Delta H\left(\varepsilon_{X}, \varepsilon_{Y}, \varepsilon_{Z}, \Delta m\right)$ (3) thay đổi ở mức rất lớn. Ví dụ, theo (Hướng dẫn sử dụng các tham số tính chuyến từ Hệ tọa độ quốc tế WGS-84 sang Hệ tọa độ quốc gia VN-2000 và ngược lại), khi chuyển các tọa độ từ hệ tọa độ quốc tế WGS-84 về Hệ tọa độ quốc gia VN2000, các góc Euler $\varepsilon_{X}=0 ", 00928836, \varepsilon_{Y}=-0 ", 01975479$ chỉ làm đại lượng $\Delta H\left(\varepsilon_{X}, \varepsilon_{Y}, \varepsilon_{Z}, \Delta m\right)$ (3) thay đổi ở mức 1-2 mm, tuy nhiên đại lượng thay đổi tỷ lệ xích $\Delta m=-0,000000252906278$ đã làm đại lượng $\Delta H\left(\varepsilon_{X}, \varepsilon_{Y}, \varepsilon_{Z}, \Delta m\right)$ (3) thay đổi đến giá trị -1,6 m.

Như vậy, nghịch lý từ sự xuất hiện các sai sô trong các tọa độ của các điểm thiên văn - trắc địa do sự tích lũy sai số của các trị đo thiên văn - trắc địa trong quá trình truyền tọa độ từ điểm gốc $\mathrm{O}$ dẫn đến sự xuất hiện các tham số chuyển tọa độ $\varepsilon_{X}, \varepsilon_{Y}, \varepsilon_{Z}, \Delta m$ và tiếp theo gây sai số đáng kể trong độ cao trắc địa quốc gia $H$ được tính chuyển từ độ cao trăc địa toàn cầu $\bar{H}$ theo công thức (2). Điều này phá vỡ quan hệ chặt chẽ giữa độ cao trắc địa toàn cầu $\bar{H}$ và độ cao trắc địa quốc gia $H$ được thiết lập bởi công thức (4) thông qua quá trình định vị ellipsoid WGS84 quy chiếu quốc gia sát nhất với mặt quasigeoid quốc gia, không những thế nó còn làm sai lệch việc chuyển độ cao quasigeoid hỗn hợp $\bar{\zeta}$ thành độ cao quasigeoid quốc gia $\zeta$ theo công thức (5) khi lưu ý rằng công thức (5) nhận được từ mối quan hệ chặt chẽ giữa ellipsoid WGS84 quy chiếu quốc gia và ellipsoid WGS84 quy chiếu quốc tế từ các kết quả định vị ellipsoid.

Mục đích của bài báo khoa học này là nghiên cứu phương pháp để loại bỏ nghịch lý được nêu ở trên.

\section{Giải quyết vấn đề}

Như đã trình bày ở trên, đại lượng thay đổi tỷ lệ xích $\Delta m$ trong 07 tham số $d X_{0}, d Y_{0}, d Z_{0}$, $\varepsilon_{X}, \varepsilon_{Y}, \varepsilon_{Z}, \Delta m$ nhận được trong quá trình bình sai mạng lưới thiên văn - trắc địa hạng I, II truyền thống với các trị đo GPS và được sử dụng để chuyển các tọa độ từ hệ tọa độ WGS84 về hệ quy chiếu quốc gia là một trong những yếu tố chủ yếu gây ra sai lệch lớn trong độ cao trắc địa quốc gia $H$ nhận 
được từ độ cao trắc địa toàn cầu $\bar{H}$ theo công thức (2). Đại lượng $\Delta m$ thường có độ lớn ở mức $10^{-7}$ do theo quy định xây dựng mạng lưới thiên văn - trắc địa, các sai số tương đối của các cạnh đáy hạng I, II ở mức $1 / 300.000$ - 1/400.000, còn các sai số tương đối của các cạnh đường chuyền hạng I, II ở mức $1 / 250.000$ - 1/300.000. Các sai số tương đối nêu trên của các cạnh trong mạng lưới thiên văn - trắc địa là khá lớn so với sai số tương đối của cạnh được xác định bằng công nghệ GNSS hiện nay.

Với mục đích giảm thiểu đại lượng thay đổi tỷ lệ xích $\Delta m$ nêu trên, khi sử dụng bổ sung các dữ liệu độ cao chuẩn hạng I, II quốc gia và GNSS chúng ta làm như sau:

- Tạo mạng lưới GNSS phủ trùm gồm $\mathrm{n}$ điểm GNSS phân bố tương đối đồng đều trên lãnh thổ quốc gia với khoảng cách trung bình từ $20-100 \mathrm{~km}$, các dữ liệu GNSS được xử lý trong ITRF tương ứng với ellipsoid WGS84 quốc tế vào thời điểm $\mathrm{t}_{0}$;

- Nếu các điểm GNSS là các điểm độ cao hạng I, II quốc gia, thì chúng ta sử dụng 07 tham số trong 07 tham số $d X_{0}, d Y_{0}, d Z_{0}, \varepsilon_{X}, \varepsilon_{Y}, \varepsilon_{Z}, \Delta m$ nhận được trong quá trình bình sai mạng lưới thiên văn - trắc địa hạng $\mathrm{I}$, II truyền thống với các trị đo GPS để xác định các tọa độ trắc địa $\mathrm{B}, \mathrm{L}$ của chúng trong hệ tọa độ phẳng quốc gia theo công thức (1). Như đã trình bày ở trên, trong trường hợp này các tham số $\varepsilon_{X}, \varepsilon_{Y}, \varepsilon_{Z}, \Delta m$ ảnh hưởng nhỏ đến các tọa độ trắc địa $\mathrm{B}, \mathrm{L}$ được tính chuyển.

Nếu các điểm GNSS là các điểm thiên văn - trắc địa hạng I, II quốc gia, thì sử dụng mô hình quasigeoid hỗn hợp độ chính xác cao để tính các độ cao quasigeoid hỗn hợp của các điểm này. Mô hình quasigeoid hỗn hợp độ chính xác cao được xây dựng dựa trên các điểm độ cao hạng I, II ổn định có đo GNSS.

- Các điểm GNSS được bố trí ở các vị trí ổn định về mặt địa chất kiến tạo, không bị tác động của các yếu tố nhân sinh, đảm bảo thông thoáng bầu trời để thu các tín hiệu GNSS và dễ tìm kiếm trên thực địa.

Các tiêu chuẩn nêu trên được áp dụng để xây dựng các điểm khống chế không gian của mạng lưới GNSS phủ trùm thuộc hệ quy chiếu không gian ở các nước, ví dụ mạng lưới HARN (High Accuracy Reference Networks - Mỹ), mạng lưới khống chế thụ động (PCN - Passive Control Networks, Canada), mạng lưới AGN (Auscope GNSS Network -Australia) v..v.

So với các điểm thiên văn - trắc địa truyền thống có một số điểm được đo GNSS, các điểm khống chế không gian của mạng lưới GNSS phủ trùm có chứa nhiều nguồn dữ liệu hơn hẳn: Tất cả các điểm khống chế không gian có chứa các độ cao chuẩn $/^{\gamma}$ hạng I, II trong hệ độ cao quốc gia, các tọa độ trắc địa $\mathrm{B}, \mathrm{L}$ (hoặc các tọa độ phẳng) trong hệ tọa độ phẳng quốc gia tương ứng với ellipsoid WGS84 quy chiếu quốc gia, các tọa độ ellipsoid $\bar{B}, \bar{L}, \bar{H}$ tương ứng với ellipsoid WGS84 quy chiếu quốc tế nhận được từ công nghê GNSS.

Đối với các điểm khống chế không gian quốc gia không phải là các điểm thiên văn - trắc địa, các tọa độ trắc địa $\mathrm{B}, \mathrm{L}$ (hoặc các tọa độ phẳng) trong hệ tọa độ phẳng quốc gia nhận được từ các

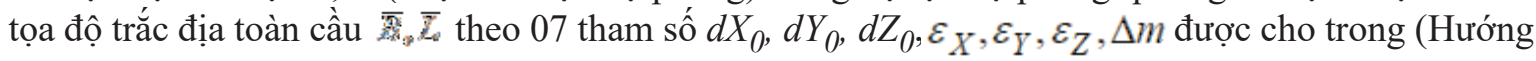
dẫn sử dụng các tham số tính chuyển từ Hệ tọa độ quốc tế WGS-84 sang Hệ tọa độ quốc gia VN2000 và ngược lại). Như đã trình bày ở trên, các tham số $\varepsilon_{X}, \varepsilon_{Y}, \varepsilon_{Z}, \Delta m$ gây ra biến thiên rất nhỏ trong các tọa độ trắc địa $\mathrm{B}, \mathrm{L}$ được tính chuyển.

Với đa dạng dữ liệu trắc địa độ chính xác cao trên điểm khống chế không gian, chúng ta có thể giải quyết nhiều bài toán để xây dựng hệ quy chiếu không gian, trong đó bài toán quan trọng nhất 
phục vụ xây dựng mô hình quasigeoid độ chính xác cao - đó là bài toán định vị lại ellipsoid WGS84 quy chiếu quốc gia dựa trên hệ phương trình (5), chỉ có khác là xác định tham số chuyển tọa độ mới

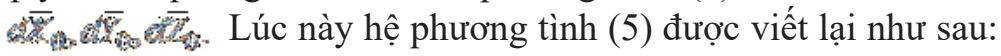

$$
\zeta=\overline{\bar{\zeta}}+A \cdot\left(\begin{array}{l}
d \bar{X}_{0} \\
d \bar{Y}_{0} \\
d \bar{Z}_{0}
\end{array}\right) .
$$

Việc định vị lại ellipsoid WGS84 quy chiếu quốc gia sát nhất với mặt quasigeoid quốc gia không chỉ cho phép nhận được các tham số chuyển tọa độ mới cách đơn trị độ cao trắc địa toàn cầu $\bar{H}$ tương ứng với ellipsoid WGS84 quy chiếu quốc tế thành độ cao trắc địa quốc gia $H$ tương ứng với ellipsoid WGS84 quy chiếu quốc gia theo công thức:

$$
H=\bar{H}+A \cdot\left(\begin{array}{l}
d \bar{X}_{0} \\
d \bar{Y}_{0} \\
d \bar{Z}_{0}
\end{array}\right) \text {. }
$$

mà còn cho phép nhận được dãy gồm $\mathrm{n}$ giá trị độ cao quasigeoid quốc gia độ chính xác cao từ các độ cao quasigeoid hỗn hợp GNSS/thủy chuẩn theo công thức (6) trên $\mathrm{n}$ điểm khống chế không gian phục vụ việc xây dựng mô hình quasigeoid quốc gia độ chính xác cao.

Như đã trình bày ở trên, các tọa độ trắc địa $\mathrm{B}, \mathrm{L}$ của các điểm khống chế không gian trong hệ tọa dộ phẳng quốc gia chịu ảnh hưởng rất nhỏ của đại lượng thay đổi tỷ lệ xích $\Delta m$ nhận được trong quá trình bình sai mạng lưới thiên văn - trắc địa hạng $\mathrm{I}$, II truyền thống với các trị đo GPS. Do đó

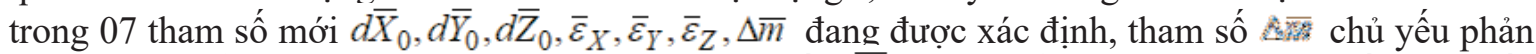
ánh sự thay đổi tỷ lệ xích của độ cao trắc địa toàn cầu $\bar{H}$ khi chuyển nó thành độ cao trắc địa quốc gia $H$ trong hệ quy chiếu không gian quốc gia theo công thức (7). Tuy nhiên trong điều kiện chưa có mô hình quasigeoid quốc gia độ chính xác cao, độ cao trắc địa quốc gia $H$ trong hệ quy chiếu không gian quốc gia lại được xác định từ độ cao trắc địa toàn cầu $\bar{H}$ theo công thức (7). Điều này có nghĩa là tham số $\mathcal{A S}^{\text {烈 }}$ phải bằng 0 . Bằng cách như vậy chúng ta sẽ loại bỏ điều nghịch lý được nêu ở trên khi xây dựng hệ quy chiếu không gian quốc gia.

Chúng ta xem xét phương pháp xác định các tham số mới $\bar{\varepsilon}_{X}, \bar{\varepsilon}_{Y}, \bar{\varepsilon}_{Z}, \Delta \bar{m}$. Khi đã biết các tọa độ không gian $\bar{X}, \bar{Y}, \bar{Z}$ của điểm khống chế không gia trong ITRF, còn các tọa độ không gian $X$, $Y, Z$ của điểm trắc địa trong hệ $\mathrm{VN} 2000$ - 3D được xác định theo công thức:

$$
\begin{aligned}
& X=(N+H) \cdot \cos B \cdot \cos L, \\
& Y=(N+H) \cdot \cos B \cdot \sin L, \\
& Z=\left[N \cdot\left(1-e^{2}\right)+H\right] \cdot \sin B .
\end{aligned}
$$

ở đây $B, L$ - các tọa độ trắc địa của điểm khống chế không gian trong hệ tọa độ phẳng quốc gia; Bán kính cong trên mặt thẳng đứng thứ nhất $N$ của điểm được xác định theo công thức $N=\frac{a}{\sqrt{1-e^{2} \cdot \sin ^{2} B}}$ độ cao trắc địa quốc gia được xác định theo công thức (7).

Do các tham số chuyển tọa độ các tham số này chúng ta lập hệ phương trình số cải chính ở dạng sau: 


$$
\begin{aligned}
& v_{X}=-\bar{Z} \cdot \bar{\varepsilon}_{Y}+\bar{Y} \cdot \bar{\varepsilon}_{Z}+\bar{X} \cdot \Delta \bar{m}+l_{X}, \\
& v_{Y}=\bar{Z} \cdot \bar{\varepsilon}_{X}-\bar{X} \cdot \bar{\varepsilon}_{Z}+\bar{Y} \cdot \Delta \bar{m}+l_{Y}, \\
& v_{Z}=-\bar{Y} \cdot \bar{\varepsilon}_{X}+\bar{X} \cdot \bar{\varepsilon}_{Y}+\bar{Z} \cdot \Delta \bar{m}+l_{Z},
\end{aligned}
$$

ở đây các số hạng tự do $l_{X}=\bar{X}+d \bar{X}_{0}-X, l_{Y}=\bar{Y}+d \bar{Y}_{0}-Y, l_{Z}=\bar{Z}+d \bar{Z}_{0}-Z$.

Từ tập hợp các điểm của mạng lưới GNSS phủ trùm chúng ta lập được hệ phương trình dạng (8). Giải hệ phương trình này dưới điều kiện $\Sigma\left(v_{X}^{2}+v_{Y}^{2}+v_{Z}^{2}\right)=$ min, chúng ta sẽ nhận được các tham số ẩn $\bar{\varepsilon}_{X}, \bar{\varepsilon}_{Y}, \bar{\varepsilon}_{Z}$ và $\Delta \bar{m}$.

Với mục đích thực nghiệm, trong tài liệu (Ha Minh Hoa, 2017) đã sử dụng mạng lưới độ cao hạng I, II quốc gia bao gồm 164 điểm độ cao ổn định phân bố tương đối đều trên lãnh thổ Việt Nam. Các dữ liệu GPS trên các điểm được xử lý trong ITRF. Các tọa độ trắc địa $\mathrm{B}, \mathrm{L}$ của các điểm trong hệ tọa độ VN2000-2D được xác định từ các tọa độ trắc địa toàn cầu tương ứng với ellipsoid WGS84 quốc tế theo 07 tham số chuyển tọa độ $d X_{0}, d Y_{0}, d Z_{0}, \varepsilon_{X}, \varepsilon_{Y}, \varepsilon_{Z}, \Delta m$ được cho trong (Hướng dẫn sử dụng các tham số tính chuyển từ Hệ tọa độ quốc tế WGS-84 sang Hệ tọa độ quốc gia VN-2000 và ngược lại).

Các tham số chuyển tọa độ mới từ ellipsoid WGS84 quốc tế về ellipsoid WGS84 quốc gia nhận được sau khi định vị lại ellipsoid WGS84 quốc gia với mục đích xây dựng hệ quy chiếu tọa độ không gian quốc gia khởi đầu (được gọi là VIGAC2017) có các giá trị như sau:

$$
\begin{aligned}
& d \bar{X}_{0}=204,511083 \mathrm{~m}, d \bar{Y}_{0}=42,192468 \mathrm{~m}, d \bar{Z}_{0}=111,417880 \mathrm{~m}, \\
& \bar{\varepsilon}_{X}<\text { radian }>=-0.000000054 ; \quad \bar{\varepsilon}_{Y}<\text { radian }>=0,000000415 ; \\
& \bar{\varepsilon}_{Z}<\text { radian }>=-0,000001941 ; \Delta \bar{m}=0 .
\end{aligned}
$$

Các kết quả thử nghiệm trên các điểm GPS thuộc các mạng lưới địa động lực miền Bắc, miền Trung và Tây Nguyên cho thấy đại lượng $\Delta H\left(\bar{\varepsilon}_{X}, \bar{\varepsilon}_{Y}, \bar{\varepsilon}_{Z}, \bar{\Delta} m\right)$ trong công thức (3) chỉ ở mức $1 \mathrm{~mm}$. Như vậy, trong thực tế các tham số $\bar{\varepsilon}_{X}, \bar{\varepsilon}_{Y}, \bar{\varepsilon}_{Z}$ và $\Delta \bar{m}$. ảnh hưởng nhỏ bỏ qua đến kết quả chuyển độ cao trắc địa toàn cầu $\bar{H}$ thành độ cao trắc địa quốc gia $H$ trong hệ quy chiếu không gian quốc gia theo công thức (2), tức công thức (7) được sử dụng khi giải quyết bài toán định vị lại ellipsoid sẽ được bảo toàn trong quá trình chuyển độ cao trắc địa toàn cầu $\bar{H}$ thành độ cao trắc địa quốc gia $H$ trong hệ quy chiếu không gian quốc gia.

Chúng ta thấy rằng việc xây dựng các điểm khống chế không gian quốc gia thuộc hệ quy chiếu không gian quốc gia theo phương pháp được trình bày ở trên cho phép loại bỏ sự nghịch lý nẩy sinh do sử dụng 07 tham số chuyển tọa độ $d X_{0}, d Y_{0}, d Z_{0}, \varepsilon_{X}, \varepsilon_{Y}, \varepsilon_{Z}, \Delta m$ được cho trong (Hướng dẫn sử dụng các tham số tính chuyển từ hệ tọa độ quốc tế WGS-84 sang Hệ tọa độ quốc gia VN-2000

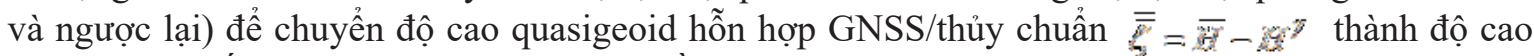
quasigeoid quốc gia $\zeta$ theo công thức (5). Bằng cách như vậy chúng ta sẽ tạo được dãy độ cao quasigeoid quốc gia trên các điểm khống chế không gian quốc gia phục vụ việc xây dựng mô hình quasigeoid quốc gia độ chính xác cao.

Nhân tiện trong bài báo này chúng ta sẽ kiểm tra sự đồng nhất của mô hình quasigeoid toàn cầu EGM2008-WGS84 trên lãnh thổ Việt Nam. Một số nhà trắc địa Việt Nam cho rằng mô hình quasi- 
geoid toàn cầu EGM2008-WGS84 không đồng nhất trên lãnh thổ Việt Nam khi vin vào cớ rằng Việt Nam thuộc nước "Fill-in", tức số liệu trọng lực ở Việt Nam không được sử dụng để xây dựng mô hình EGM2008. Thực tế không phải như vậy. Ở miền Nam Việt Nam, trong giai đoạn 1960 - 1961, các chuyên gia Mỹ (Whalen C.T., Schweninger J.B., Schoppand J.H.) đã xây dựng điểm gốc trọng lực tại sân bay Sài Gòn và liên kết với hệ thống trọng lực quốc tế Potsdam (1909) qua điểm ToKyo (Nhật Bản) và điểm SinhGapor nhờ máy L \& R.G (Lacoste and Romberg Gravity - meters). Từ điểm gốc này trong giai đoạn 1962 - 1964 đã tiến hành đo được 18 điểm hạng I là Tân An, Mỹ Tho, Vĩnh Long, Sa Đéc, Rạch Giá, Long Xuyên, Bạc Liêu, Châu Đốc, Cần Thơ, Sài Gòn, Đà Lạt AP, Đà Lạt (NGS), Nha Trang, Đà Nẵng, Quy Nhơn, Huế, Buôn Mê Thuộc, PleiKu. Độ chính xác ở mức $\pm(0,03$ - 0,07) mGal. Trong những năm 1973 - 1979, trên vùng biển Việt Nam, nhiều công ty dầu khí của các nước Anh, Pháp, Italia, Na Uy, Liên Xô (cũ) đã tiến hành khảo sát địa vật lý theo các lô trên các cấu tạo được dự đoán là triển vọng có dầu và trên toàn bộ thềm lục địa Việt Nam. Vào những năm 1980, các tàu Poisk, Iskatel, Gambuxep, Malugin đã tiến hành khảo sát địa vật lý, chủ yếu là địa chấn trên một số cấu tạo có triển vọng như Bạch Hổ, Rồng, Đại Hùng. Các dữ liệu trọng lực, từ và địa chấn nông trên vùng thềm lục địa miền Trung và Đông Nam nước ta đã được tàu Attalance (Pháp) đo năm 1993.

Trong tài liệu (Pavlis Nikolas K, Simon A. Holmes, Steve C. Kenyon and John K. Factor, 2012) đã thông báo rằng khi xây dựng mô hình trọng trường trái đất EGM2008 đã sử dụng các hệ số điều hòa cầu từ mô hình trọng trường trái đất chỉ từ vệ tinh $\mathrm{ITG}$ - GRACE03S với bậc khai triển điều hòa 180 của Viện Trắc địa lý thuyết (thuộc trường Đại học Bonn, CHLB Đức), thêm vào đó mô hình này được xây dựng dựa trên dự án vệ tinh GRACE. Dữ liệu trọng lực từ dự án vệ tinh GRACE đã bao phủ lãnh thổ Việt Nam. Trên các đại dương đã sử dụng các giá trị dị thường trọng lực được xác định bằng phương pháp altimetry từ các dự án vệ tinh altimetry TOPEX, ERS-1, GEOSAT do các tổ chức DNSC (Đan Mạch) và SIO/NOAA (Mỹ) xác định cùng với việc xây dựng mô hình MDT tương ứng (Steve Kenyon, John Factor, Nikolas Pavlis, Simon Holmes, 2007; Nikolas K. Pavlis, Simon A. Holmes, Steve C. Kenyon, John K. Factor, 2008).

Như vậy không thể nói được là các dữ liệu trọng trường được các tổ chức quốc tế thu thập được trên lãnh thổ Việt Nam không được sử dụng để xây dựng mô hình EGM2008. Chỉ có các số liệu đo trọng lực chi tiết do người Việt Nam thực hiện là không được sử dụng để xây dựng mô hình EGM2008. Khi chuyển độ cao quasigeoid toàn cầu EGM2008-WGS84 về mặt quasigeoid Hòn Dấu, chúng ta được mô hình quasigeoid hỗn hợp VIGAC2014 với độ chính xác $\pm 0,07$ m Hà Minh Hòa, và nnk, 2012; Hà Minh Hòa, Nguyễn Bá Thủy, Phan Trọng Trịnh, Nguyễn Nguyên Cương và nnk, 2016). Vậy mô hình VIGAC2014 có đồng nhất trên lãnh thổ Việt nam hay không ?. Chúng ta sẽ kiểm ta điều này. Do độ cao quasigeoid $\overline{\bar{\zeta}}^{*}$ từ mô hình VIGAC2014 là độ cao quasigeoid hỗn hợp, nên từ (6) suy ra công thức chuyển cao quasigeoid $\bar{\zeta}^{*}$ thành độ cao quasigeoid quốc gia $\zeta^{*}$ ở dạng sau:

$$
\xi^{*}=\bar{\zeta}^{*}+A \cdot\left(\begin{array}{l}
d \bar{X}_{0} \\
d \bar{y}_{0} \\
d \bar{Z}_{0}
\end{array}\right),
$$

ở đây $\overline{\bar{\zeta}}^{*}=\bar{\zeta}+0,890 m, \bar{\zeta}$ - độ cao quasigeoid toàn cầu EGM2008-WGS84.

Như vậy trên 164 điểm độ cao hạng I, II ổn định, được phân bố tương đối đồng đều trên lãnh thổ Việt Nam chúng ta có hai dãy độ cao quasigeoid quốc gia hoàn toàn độc lập nhau: 
- Dãy độ cao quasigeoid quốc gia $\zeta$ nhận được từ kết quả định vị lại ellipsoid quốc gia được tính theo công thức (6) với các tham số $d \bar{X}_{0}, d \bar{Y}_{0}, d \bar{Z}_{0}$ ược cho trong (9) và $\overline{\bar{\zeta}}$ là độ cao quasigeoid hỗn hợp GPS/thủy chuẩn;

- Dãy độ cao quasigeoid quốc gia $\zeta^{*}$ nhận được theo công thức (10) với các tham số $d \bar{X}_{0}, d \bar{Y}_{0}, d \bar{Z}_{0}$ được cho trong (9).

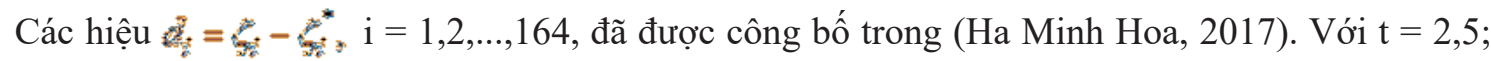

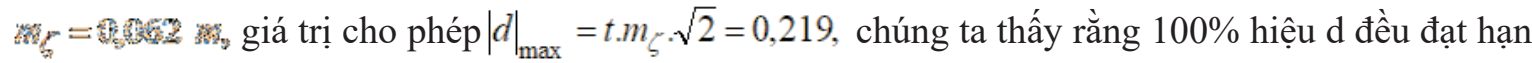
sai, hiệu lớn nhất bằng $0,195 \mathrm{~m}$ thuộc mốc độ cao IVL-HT73. Như vậy có thể nói rằng mô hình quasigeoid toàn cầu EGM2008-WGS84 phân bố đồng nhất trên lãnh thổ Việt Nam, mặc dù độ chính xác của mô hình chưa cao hơn $\pm 0,04 \mathrm{~m}$ theo yêu cầu.

Việc sử dụng hai dãy độ cao quasigeoid quốc gia độc lập nêu trên để xây dựng mô hình quasigeoid quốc gia khởi đầu VIGAC2017 đã được trình bày trong (Ha Minh Hoa, 2018).

\section{Kết luận}

Việc xác định 07 tham số chuyển tọa độ $d X_{0}, d Y_{0}, d Z_{0}, \varepsilon_{X}, \varepsilon_{Y}, \varepsilon_{Z}, \Delta m$ từ ellipsoid WGS84 quốc tế về ellipsoid WGS84 quốc gia nhờ việc bình sai mạng lưới thiên văn - trắc địa hạng I, II truyền thống kết hợp với các trị đo GPS trong hệ tọa độ phẳng quốc gia không cho phép chuyển đổi chính xác độ cao trắc địa toàn cầu từ ellipsoid WGS84 quốc tế về ellipsoid WGS84 quốc gia. Nguyên nhân là do trong mạng lưới thiên văn - trắc địa hạng I, II truyền thống, độ chính xác đo các cạnh trong các lưới đường truyền hạng I, II không cao so với độ chính xác tính toán các chiều dài cạnh nhờ công nghệ GNSS. Do đó đại lượng thay đổi tỷ lệ xích $\Delta m$ giữa ITRF và hệ tọa độ phẳng quốc gia là khá lớn ở mức $10^{-7}$. Khi xây dựng hệ quy chiếu không gian, đại lượng thay đổi tỷ lệ xích $\Delta m$ sẽ xấp xỉ bằng 0 . Điều này đảm bảo chuyển đổi chính xác cao độ cao trắc địa toàn cầu từ ellipsoid WGS84 quốc tế về ellipsoid WGS84 quốc gia. Đó là cơ sở xác định độ cao quasigeoid độ chính xác cao trên các điểm khống chế không gian quốc gia phục vụ việc xây dựng mô hình quasigeoid quốc gia độ chính xác cao./.O

\section{Tài liệu tham khảo}

[1]. Hướng dẫn sử dụng các tham số tính chuyển từ Hệ tọa độ quốc tế WGS-84 sang Hệ tọa độ quốc gia VN-2000 và ngược lại. Công văn số 1123/ĐĐBĐ-CNTĐ ngày 26/10/2007 của Cục Đo đạc và Bản đồ Việt Nam.

[2]. Hà Minh Hòa, Nguyễn Ngọc Lâu, 2013. Nguyên lý lý thuyết và thực tiễn của Trắc địa vũ trụ. Nhà xuất bản Khoa học và Kỹ thuật, 276 trg., Hà Nội - 2013 .

[3]. Hà Minh Hòa, và nnk, 2012. Nghiên cứu cơ sở khoa học của việc hoàn thiện hệ độ cao gắn liền với việc xây dựng hệ tọa độ động lực quốc gia, 247 trg.. Đề tài khoa học và công nghệ cáp Bộ Tài nguyên và Môi trường giai đoạn 2010 - 2012. Hà Nội - 2012.

[4]. Hà Minh Hòa, Nguyễn Bá Thủy, Phan Trọng Trịnh, Nguyễn Nguyên Cương và nnk, 2016. "Nghiên cứu đánh giá các mặt chuẩn mực nước biển (mặt "0" độ sâu, trung bình và cao nhất) theo các phương pháp trắc địa, hải văn và kiến tạo hiện đại phục vụ xây dựng các công trình và quy hoạch đới bờ Việt Nam trong xu thế biến đổi khí hậu", 563 trg.. Đề tài khoa học và phát triển công nghệ mã số KC.09.19/11 -15 giai đoạn 2012 - 2015 thuộc Chương trình KH\&CN trọng điểm cấp Nhà nước KC-09/11-15 "Nghiên cứu khoa học và công nghệ phục vụ quản lý biển, hải đảo và phát triển 
kinh tế biến” giai đoạn 2011 - 2015. Bộ Khoa học và Công nghệ, Hà Nội - 2016.

[5]. Ha Minh Hoa, 2017. Construction of inital national quasigeoid model VIGAC2017, First step to national spatial reference system in Vietnam. Vietnam journal of Earth Sciences, 39 (2): 155 166 ,

DOI: 10.15625/0866-7187/39/2/9702, Vietnam Academy of Science and Technology, http://www.vjs.ac.vn/index.php/jse.

[6]. Ha Minh Hoa, 2018. Improvement of the accuracy of the quasigeoid model VIGAC2017. Vietnam journal of Earth Sciences, 40 (1): 38 - 45, DOI: 10.15625/0866-7187/40/1/10914, Vietnam Academy of Science and Technology, http://www.vjs.ac.vn/index.php/jse.

[7]. Nikolas K. Pavlis, Simon A. Holmes, Steve C. Kenyon, John K. Factor, 2008. An Earth gravitational model to degree 2160: EGM2008. EGU General asembly 2008, Vienna, Austria, April 13 18,2008

[8]. Pavlis Nikolas K, Simon A. Holmes, Steve C. Kenyon and John K. Factor, 2012. The development amd evaluation of the Earth gravitational model (EGM2008). Journal of Geophisical Research, Vo. 117, B04406, 1-38, doi: 10.1029/2011JB008916

[9]. Steve Kenyon, John Factor, Nikolas Pavlis, Simon Holmes, 2007. Towards The Next Earth Gravitational Model. 2007 SEG Annual Meeting, 23-28 September, 2007, San Antonio, Texas. SEG2007-0733, Society of Exploration Geophisicits. $\bigcirc$

\section{Summary}

Improvement of the accuracy of the national quasigeoid model based on construction of the national spatial reference system

Ha Minh Hoa

Vietnam Institute of Geodesy and Cartography

In a process of construcion of the national spatial reference system, based on a reorientation of the WGS84 national reference ellipsoid with purpose of the best it's fitting to the national quasigeoid, we will can increase the accuracy of the seven parameters of coordinate conversion from the ITRF to the national spatial reference system, especially of the scale change parameter between two above mentioned coordinate systems. That allows us to accurately convert the global geodetic height determined by GNSS technology from the WGS 84 to the national spatial reference system serving a construction of the highly accurate national quasigeoid model. This scientific article will prove a correctness of this approach. $\bigcirc$ 